\title{
Ball-pen probe diagnostics of a weakly magnetized discharge plasma column
}

\author{
L. Šalamon, ${ }^{1}$ G. Ikovic, ${ }^{1}$ T. Gyergyek, ${ }^{3,2}$ J. Kovačič ${ }^{* 2}$ and B. Fonda ${ }^{1}$ \\ ${ }^{1}$ University of Ljubljana, Faculty of Mathematics and Physics \\ Ljubljana, Slovenia \\ ${ }^{2}$ Institut 'Jožef Stefan' \\ Ljubljana, Slovenia \\ ${ }^{3}$ University of Ljubljana, Faculty of electrical engineering \\ Ljubljana, Slovenia \\ E-mail: jernej.kovaciceijs.si
}

\begin{abstract}
The ball-pen probe is an electric probe developed for direct measurements of plasma potential in magnetized plasmas. It has been succesfully tested and used in tokamak plasmas, while its behaviour in low-temperature magnetized plasma is still subject to investigation. The ball-pen probe can adjust the ratio of electron and ion saturation current $I_{\text {sat }}^{-} / I_{\text {sat }}^{+}$to be close to 1 . Therfore its current-voltage characteristic becomes symetric and floating potential of the probe goes towards the plasma potential in Maxwellian plasma. Study of ball-pen probe operation in linear magnetized plasma device in Ljubljana is made at different plasma conditions for different probe designs.
\end{abstract}

First EPs Conference on Plasma Diagnostics - 1st ECPD,

14-17 April 2015

Villa Mondragone, Frascati (Rome) Italy

* Speaker. 


\section{Introduction}

One of the simplest and most widely used probes in low temperature plasma is the Langmuir probe (LP). It is constructed of a simple and small collector, most commonly from a cylindrical piece of a wire. From the post processing of current- potential(I-V) curve, the temperature of electrons $T_{e}$, the number density of electrons in unperturbed plasma $n_{e}$ and plasma potential $\phi_{p l}$ can be obtained. For plasma potential determination, the following simplistic relation can be used in Maxwellian plasmas:

$$
\phi_{p l}=V_{f l}-\left(\frac{k_{B} T_{e}}{e_{0}}\right) \ln R,
$$

where $V_{f l}$ is the floating potential and $R=\frac{I_{s t}^{-}}{I_{s a t}^{+}}$represents the ratio between the electron and ion saturation current. Apart from the Langmuir probe, the retarding field analyser and Katsumata probe [1] have also been used for indirect plasma potential measurements. Katsumuta probe belongs to the category of ion sensitive probes and is built from a retractable metallic collector with a flat tip, shielded inside the metallic tube. The tube axis is orientated perpendicular to the magnetic field lines in plasma. The probe operation is based on the smaller gyroradius of electrons comparing to ions in the presence of magnetic field. Therefore, when the collector is retracted inside the tube, mainly electrons are shielded. However, the electrons can not be completely screened off by the shielding tube due to the presence of $E \times B$ drift inside to the tube [3]. For this reason, the shielding tube must be biased to the same voltage as the collector to prevent the electron collection and to measure pure ion current [2].

Direct measurements of the plasma potential in magnetized plasmas can be achieved using different kind of probes, such as the emissive probe [7, 8], the plug probe [4], the tunnel probe [5], the baffle probe [6] or the Ball-pen probe [9], which will be studied in this article.

Ball-pen probe (BPP) has been developed with a possibility of direct measurements of $\phi_{p l}$, as a modified Katsumata probe. It also has a retractable collector inside the shielding tube, which is positioned perpendicular to the magnetic field lines. However, the shielding tube is made from ceramic and therefore it does not need to be biased. Idea of BPP is to reduce $I_{\text {sat }}^{-}$until it reaches $I_{s a t}^{+}$. Thus $\phi_{p l}$ approaches to $V_{f l}$ and plasma potential can be measured directly. Extracted collector with a conical tip works as a classical Langmuir probe and when retracted inside, mainly $I_{\text {sat }}^{-}$is screened off until at some depth it decreases down to $I_{\text {sat }}^{+}[10,11]$. Ball-pen probe has already been extensively tested and used in tokamak plasmas, however not many studies had been done on the behaviour of a BPP in low-temperature plasmas. In both cases the electron energy distribution function (EEDF) and the ion energy distribution function (IEDF) can be far from Maxwellian and this has not yet been thoroughly researched.

\section{Experimental setup}

Our experiment was performed in a linear magnetized plasma device (LMPD) composed of a stainless steel tube $1.5 \mathrm{~m}$ long and $17 \mathrm{~cm}$ inner diameter. Plasma is confined with solenoid magnetic field coils, which can create axial magnetic field up to $0.4 \mathrm{~T}$. The electron source is hot tungsten filamentary cathode biased to a discharge voltage $U_{d}$ between $-30 \mathrm{~V}$ and $-60 \mathrm{~V}$. The usual 
discharge current is kept between $0.5 \mathrm{~A}$ and $2 \mathrm{~A}$. Measurements were performed in argon plasma in a range of gas pressures between $1.2 \times 10^{-4}-1.8 \times 10^{-3}$ mbar at magnetic field density 10 $\mathrm{mT}$. Probe is inserted from the side, perpendicular to magnetic field lines, and is manually radially

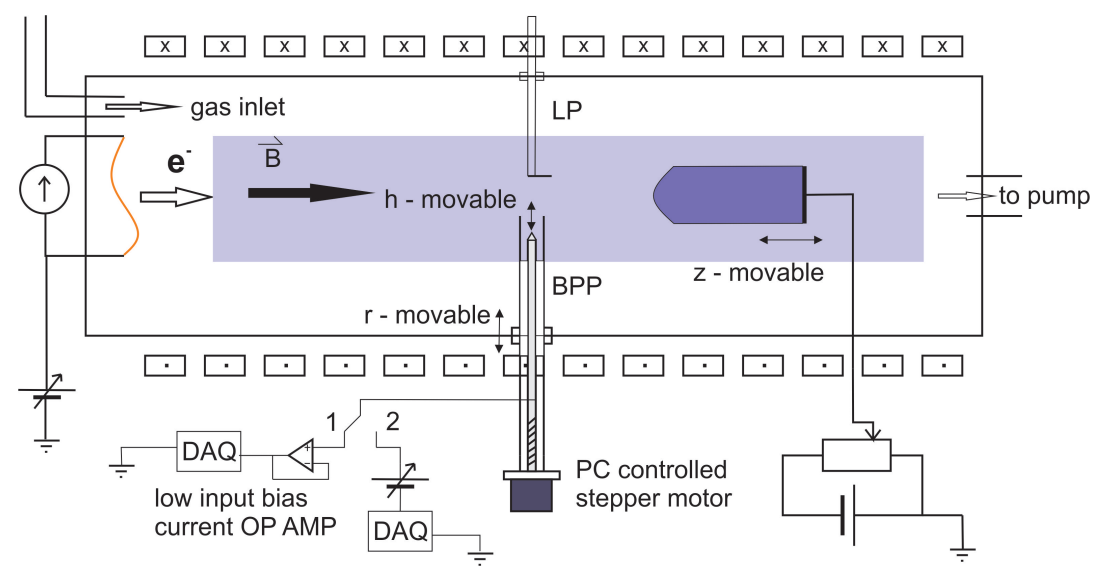

Figure 1: The experimental setup at the LMPD.

movable. The position of the collector inside the insulator tube is set by a computer controlled stepper motor. For $h>0$ the collector is partly extracted from insulator tube, thus, working as a LP and I-V probe trace can be obtained. When $h=0$, the conical tip of collector is just at the entrance of a shielding tube and for $h<0$ it is inside the tube. For each measured plasma condition I-V characteristic and floating potential versus collector position have been measured. Some had been verified using a normal LP. Three collectors and shielding tubes of different sizes were used for measurements. First, ball-pen probe with the stainless steel collector of diameter $d_{1}=1.5 \mathrm{~mm}$ and inner diameter of tube $D_{1}=2 \mathrm{~mm}$ was used. Other two designs feautured tungsten collector with dimensions $d_{2}=0.4 \mathrm{~mm}, D_{2}=0.55 \mathrm{~mm}$ and $d_{3}=0.2 \mathrm{~mm}, D_{3}=0.3 \mathrm{~mm}$. Resulting high impedance between probe collector and plasma proved to be a problem of its own. For this reason, a high input impedance electrometer was used for floating potential measurements.

\section{Experimental results}

We investigated the dependence of $V_{f l}$ of the BPP on the depth of collector insertion. The aim of our experiment was to examine the ball-pen probe operation for different EEDFs and IEDFs, which was achieved through variations in working gas pressure, selection in gases, discharge voltages, geometries of the probe and the presence of strong double layers. Some of the results are presented in this paper.

First, the largest BPP $\left(d_{1}\right.$ and $\left.D_{1}\right)$ was placed at fixed radial position in the center of plasma column. Measurements were performed for pressures between $1.2 \times 10^{-4}-1.8 \times 10^{-3}$ mbar at magnetic field $B=10 \mathrm{mT}$, discharge current $1 \mathrm{~A}$ and discharge voltage $50 \mathrm{~V}$. Low pressure provides a high energy tail of the EEDF. Corresponding electron temperatures and densities obtained from the I-V characteristics are plotted in Fig. 2. Plasma potential $\phi_{p l}$ is obtained from the maximum of the first derivative of I-V characteristic measured with BPP working as Langmuir probe at $h=5$ mm (verified with classic LP), Fig. 3. 

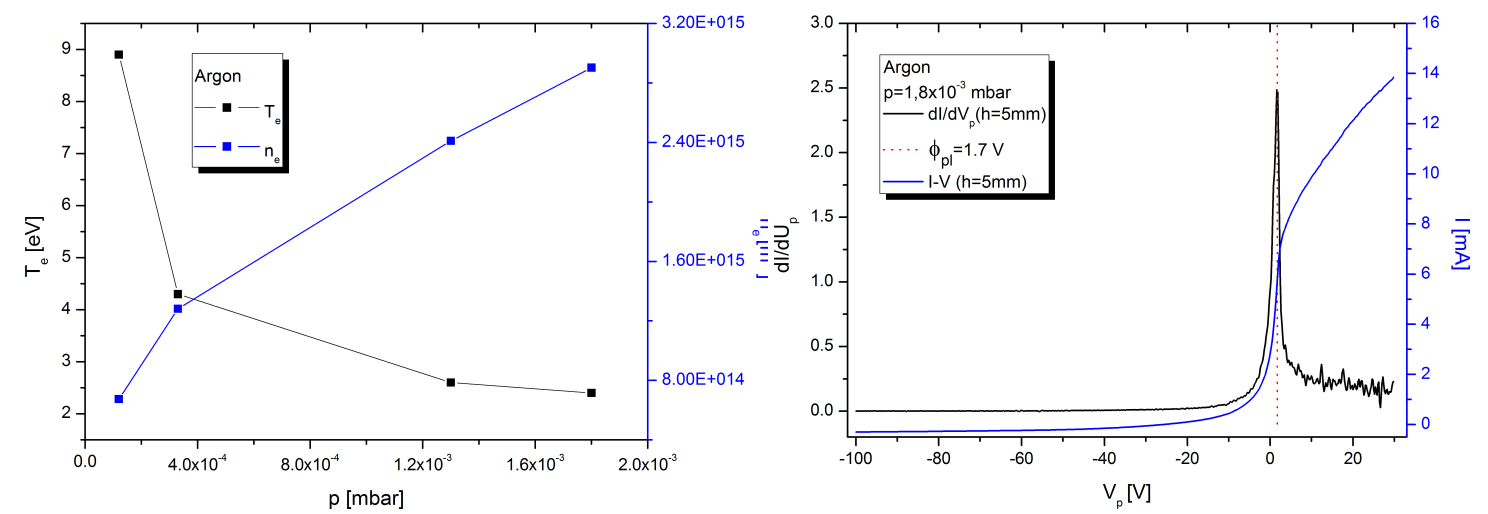

Figure 2: Electron density $n_{e}$ and electron temperature $T_{e}$ with respect to the plasma pressure for argon plasma at $B=10 \mathrm{mT}$.

Figure 3: I-V characteristic of the BPP at $h=5 \mathrm{~mm}$ and its corresponding first derivative for the argon plasma at $p=1.8 \times 10^{-3}$ mbar and $B=10 \mathrm{mT}$.

In the Fig. 4, dependency of the floating potential $V_{f l, B P P}$ of BPP on the depth of collector $\mathrm{h}$ is plotted for various pressures. The saturation of the $V_{f l, B P P}$ occurs at relatively large collector retraction $h \approx 12 \mathrm{~mm}$ due to a relatively weak magnetic field. In the Fig. 5, a graph is made showing the discrepancy between the plasma $\phi_{p l}$ and $V_{f l, B P P}$, normalized to the electron temperature. The dicrepancy proves to be larger at lower pressures where a high energy tail of the EEDF is present. The difference is getting smaller with rising the pressure as the temperature is lower and more electrons are in body of the EEDF. Thus, agreement with eq. 1.1 is better. Fig. 4 also shows a peculiarity already commented in [11], where the curve of a retracting collector's $V_{f l, B P P}$ provides a local maximum around $h=0 \mathrm{~mm}$ and more inward a distinct local minimum. We speculate that this is due to the large pitch of the ions, which is much larger than the diameter of the probe hole. We could see from the I-V curves obtained that it is actually the ion current to the collector that decreases more so than the electron part. High energy electrons with large enough gyroradius can penetrate a few $\mathrm{mm}$ into the tube, lowering the $V_{f l, B P P}$, they, nonetheless, pitch comparable to the diameter of the probe entrance.

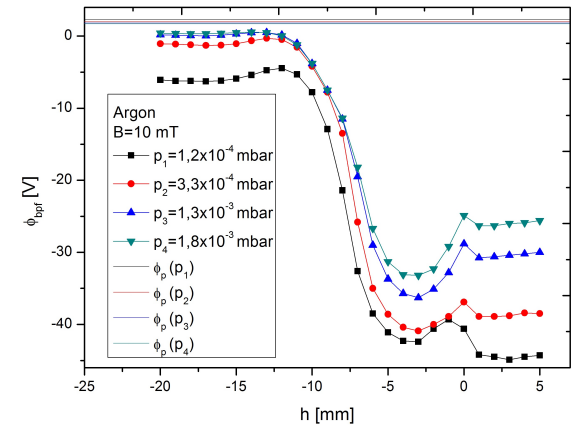

Figure 4: Dependence of a ball-pen floating potential $V_{f l, B P P}$ on the depth of collector measured in a range of pressures.

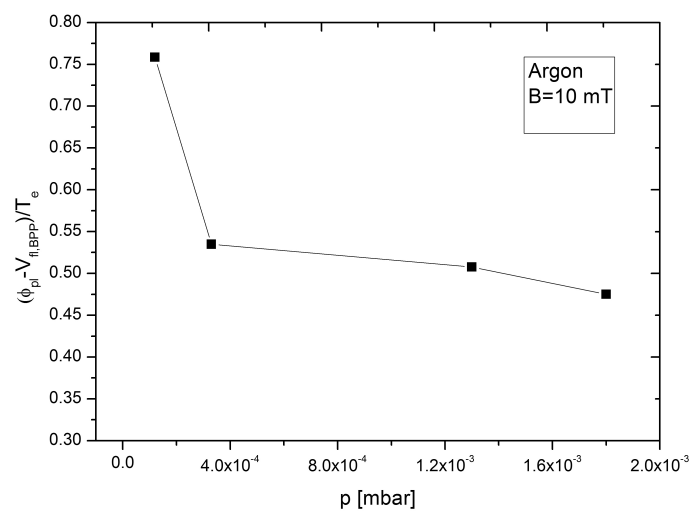

Figure 5: Dependence of the normalized difference $\left(\phi_{p l}-V_{f l, B P P}\right) / T_{e}$ with the pressure at magnetic field $B=10 \mathrm{mT}$. 
Another set of measurements was made with BPP of dimensions $d_{1}$ and $D_{1}$ at $B=10 \mathrm{mT}$ and $p=1.3 \times 10^{-3}$ varying discharge voltage $U_{d}$ between $30-60 \mathrm{~V}$. Graph of $V_{f l, B P P}$ depending on the depth of collector $\mathrm{h}$ is shown in Fig. 6. Again, local minimum of the $V_{f l, B P P}$ appears at around $h=-3 \mathrm{~mm}$. Drop of potential is lower for smaller discharge voltages. Discharge voltage defines the energy of primary electrons, which subsequently, at lower pressures, provides a tail of the EEDF with different maximum energy. The curve minimum developed proves its dependancy on the fast electrons.

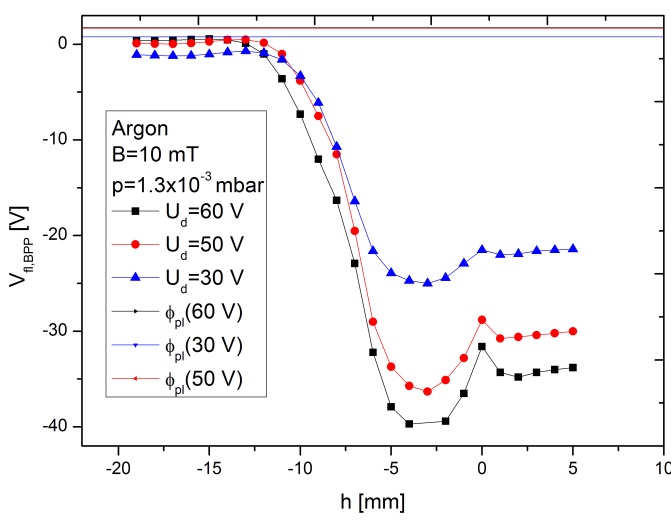

Figure 6: Dependence of a ball-pen floating potential $V_{f l, B P P}$ on the depth of collector at different discharge voltages for $D_{1}$ tube.

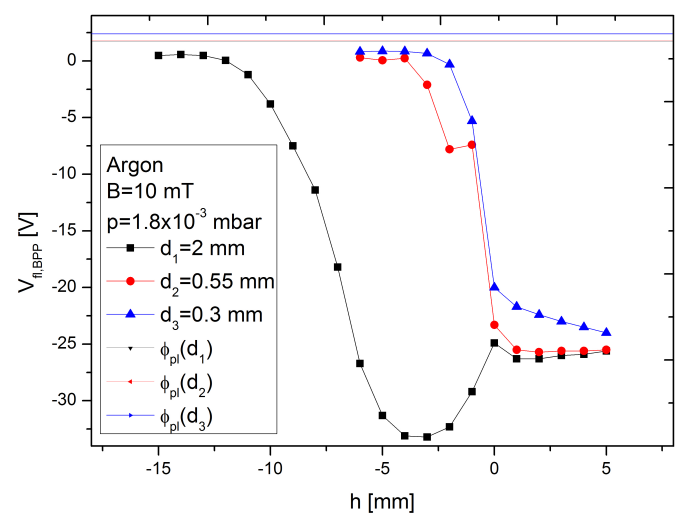

Figure 7: Examples of the ball-pen floating potential $V_{f l, B P P}$ with respect to the depth of collector for tube diameters $D_{1}, D_{2}$ and $D_{3}$. at $p=1.8 \times 10^{-3}$ and $B=10 \mathrm{mT}$.

Comparison of the floating potential of BPP with respect to the collector insertion was made for three different sizes of collector and inner diameter of tube, following [11], at same discharge parameters: $B=10 \mathrm{mT}, p=1.8 \times 10^{-3} \mathrm{mbar}, U_{d}=50 \mathrm{~V}$ and $I_{d}=1 \mathrm{~A}$. Graph for all three sizes is presented in Fig. 7. It is clearly seen that there is an influence of the tube diameter on the BPP floating potential. For $D_{2}$ and $D_{3}$ the saturation plateu is reached around $h=-4 \mathrm{~mm}$, while for tube diameter $D_{3}$ at around $h=-13 \mathrm{~mm}$. Local minimum is reduced to much smaller h range for $D_{2}$ and is not observed at measured depths for $D_{3}$. Significant jump towards the $\phi_{p l}$ is seen for BPPs with smaller tube diameters as soon as collector is located inside the tube. All of the probes are likely to measure the same saturation $V_{f l, B P P}$ value that is close to the plasma potential.

We also used the BPP for measurements in vicinity of a strong double layer (DL) formed due to an aditional anode in the plasma. In case of a DL, electrons are accelerated into the anode plasma through the potential drop comparable to the anode voltage, roughly $V_{a}=+30 \mathrm{~V}$. In Fig. 9, BPP radial measurements of $V_{f l, B P P}$ were made. The anode plasma lies between $0 \leq r \leq 5 \mathrm{~mm}$ and $0 \leq z \leq 60 \mathrm{~mm}$. Here we noticed for the first time, that there is a great difference between the $V_{f l, B P P}$ curve made when collector is moving inwards or when it is moving outwards. We can see in Fig. 8, that the saturation of the $V_{f l, B P P}$ only happens when the collector is moving inwards. If we start measurements deep inside the tube, while first grounding the collector, the $V_{f l, B P P}$ only reaches the "correct" value, when retraction is comparable to the ion gyroradius. We repeated the measurements at different radial positions at, e.g. $z=20 \mathrm{~mm}$. We can see that discrepancy between the $V_{f l, B P P}$ and the $\phi_{p l}$ measured by the LP is comparable to the case of cathode plasma. This is 


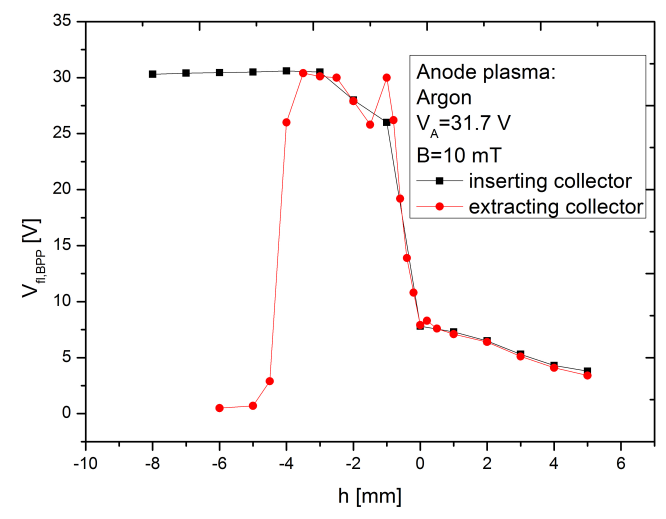

Figure 8: Floating potential of the BPP for the case of probe moving outwwards or inwards inside anode plasma.

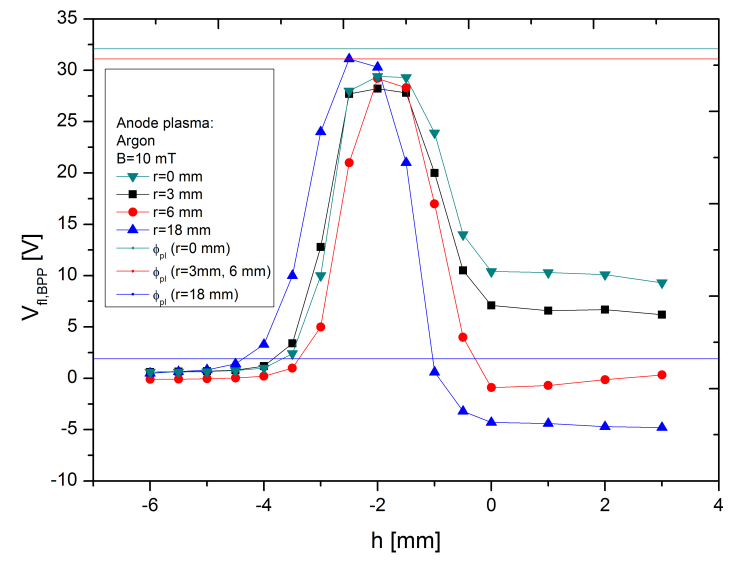

Figure 9: Floating potential of BPP at four different radial positions in or next to the anode plasma.

however not true in case of $r=18 \mathrm{~mm}$, where the collector still gathers the fast ions accelerated through DL.

\section{Summary and conclusions}

A ball-pen probe has been used for direct measurements of plasma potential in linear magnetized device in Ljubljana. Design of this probe allows us to reduce the electron saturation current to the level of ion saturation current by retracting the collector inside the insulator tube and when these saturation currents equal, the floating potential goes to plasma potential. The probe was employed to measure $\phi_{p l}$ at magnetic field $B=10 \mathrm{mT}$, pressures between $1.2 \times 10^{-4}-1.8 \times 10^{-3}$ mbar and discharge voltages between $30-60 \mathrm{~V}$ for different dimensions of the probe. For $V_{f l, B P P}$ measurements at different $h$ positions of the collector, local minium was observed when the collector was just inside the tube. Possible explanation of this phenomena could be given by a combination of different effects. We assume the highest contribution for the potential drop is due to the high energy electrons from the EEDF tail. Another contributions could be due to the $E \times B$ drift and the pitch of the ion gyroradius motion. We experienced that the electron screening is much more efficient for shielding tubes of smaller diameters, as the $V_{f l, B P P}$ drop was observed over much smaller range of $\mathrm{h}$ or was not visible at measured $\mathrm{h}$ positions. What is more, for small diameter tubes $V_{f l, B P P}$ approaches close to plasma potential at much smaller depths. Therefore, the geometry of BPP plays an important role on plasma potential measurements. We have also observed a difference depending if the measurements of the $V_{f l, B P P}$ where made when the probe was moving inward or outward. We also made measurements in the vicinity of a strong double layer where discrepany for $V_{f l, B P P}$ vs. $\phi_{p l}$ was large for cases when DL accelerated ions where collected by probe far away from the anode plasma. We learned of the importance of high input impedance circuit requirement for a succesful measurements of plasma potential as well. 


\section{References}

[1] I. Katsumata, and M. Okazaki, Ion Sensitive Probe-A New Diagnostic Method for Plasma in Magnetic fields, Jpn. J. Appl. Phys., Vol. 6, pp. 123, (1967).

[2] J. Adamek, M. Kocan, R. Panek, J. P. Gunn, E. Martines, J. St?ockel, C. Ionita, G. Popa, C. Costin, J. Brotankova, R. Schrittwieser, and G. Van Oost, Simultaneous Measurements of Ion Temperature by Segmented Tunnel and Katsumata Probe, Contrib. Plasma Phys. Vol. 48, pp. 395, (2008).

[3] D. Brunner, B. LaBombard, R. Ochoukov, R. Sullivan, and D. Whyte, Space-charge limits of ion sensitive probes, Plasma Phys. Controlled Fusion, Vol. 55, 125004, (2013).

[4] S. V. Ratynskaia, V. I. Demidov, and K. Rypdal, A probe for measurements of electrostatic fluctuations in a low-temperature magnetized plasma, Rev. Sci. Instrum., Vol. 71, 1367, (2000).

[5] J. Gunn, Measurement of Electron Temperature Fluctuations with Tunnel Probe in the CASTOR Tokamak, Phys. Plasmas, Vol. 8, 1040, (2001).

[6] V. Demidov, S. Finnegan, M. Koepke, and E. Reynolds, Baffled probe for real-time measurement of space potential in magnetized plasma, Rev. Sci. Instrum., Vol. 74, 4558, (2003).

[7] R. Schrittwieser, C. Ionita, P. Balan, C. Silva, H. Figueiredo, C. Varandas, J. J. Rasmussen, and V. Naulin, Turbulence and transport measurements with cold and emissive probes in ISTTOK, Plasma Phys. Controlled Fusion, Vol. 50, 055004, (2008). (2008).

[8] J. Sheehan, Y. Raitses, N. Hershkowitz, I. Kaganovich, and N. Fisch, A comparison of emissive probe techniques for electric potential measurements in a complex plasma, Phys. Plasmas Vol. 18, 073501 (2011).

[9] J. Adamek et al., Direct measurements of the plasma potential in ELMy H-mode plasma with ball-pen probes on ASDEX Upgrade tokamak, J. Nucl. Materials, Vol. 390-91, pp. 1114-1117, (2009).

[10] J. Adámek, M. Peterka, T. Gyergyek, P. Kudrna, and Milan Tichý, Diagnostics of magnetized low temperature plasma by ball-pen probe, Nukleonika, Vol. 1178(2) pp. 297-300, (2012).

[11] G. Bousselin, J. Cavalier, J. F. Pautex, S. Heuraux, N. Lemoine, and G. Bonhomme, Design and validation of the ball-pen probe for measurements in a low-temperature magnetized plasma, Review of Scientific Instruments, Vol. 84, 013505, (2013).

[12] V. Godyak, R. Piejak, and B. Alexandrovich, Measurements of electron energy distribution in low-pressure RF discharges, Plasma Sources Sci Technol, 1:36-58 (1992) 